This is the pre-peer reviewed version of the following article: Häbel, H., Särkkä, A., Rudemo, M., Hamngren Blomqvist, C., Olsson, E., Abrahamsson, C., and Nordin, M. (2016). From static micrographs to particle aggregation dynamics in three dimensions. Journal of Microscopy, 262(1), 102-111. doi:10.1111/jmi.12349, which has been published in final form at http://dx.doi.org/10.1111/jmi.12349. This article may be used for non-commercial purposes in accordance with Wiley Terms and Conditions for SelfArchiving. 


\section{From static micrographs to particle aggregation dynamics in three dimensions}

Henrike Häbel*1,5, Aila Särkkä ${ }^{1,5}$, Mats Rudemo ${ }^{1}$, Charlotte Hamngren Blomqvist $^{2,5}$, Eva Olsson ${ }^{2,5}$, Christoffer Abrahamsson ${ }^{3,5}$, and Matias Nordin 4

${ }^{1}$ Chalmers University of Technology and University of Gothenburg, Department of Mathematical Sciences, SE-412 96 Gothenburg, Sweden

${ }^{2}$ Chalmers University of Technology, Department of Applied Physics, SE-412 96 Gothenburg, Sweden

${ }^{3}$ Chalmers University of Technology, Department of Chemical and Biological Engineering, Applied Surface Chemistry, SE-412 96 Gothenburg, Sweden ${ }^{4}$ Stanford University, Department of Geophysics, Stanford CA 94305-2210, USA

${ }^{5}$ SuMo BIOMATERIALS, VINN Excellence Center, Chalmers University of Technology, SE-41296 Gothenburg, Sweden

April 27, 2015 


\begin{abstract}
Studies on colloidal aggregation have brought forth theories on stability of colloidal gels and models for aggregation dynamics. Still, a complete link between developed frameworks and obtained laboratory observations has to be found. In this work, aggregates of silica nanoparticles $(20 \mathrm{~nm})$ are studied using diffusion limited cluster aggregation (DLCA) and reaction limited cluster aggregation (RLCA) models. These processes are driven by the probability of particles to aggregate upon collision. This probability of aggregation is one in the DLCA and close to zero in the RLCA process. We show how to study the probability of aggregation from static micrographs on the example of a silica nanoparticle gel at $9 \mathrm{wt} \%$. The analysis includes common summary functions from spatial statistics, namely the empty space function and Ripley's $K$-function, as well as two newly developed summary functions for cluster analysis based on graph theory. One of the new cluster analysis functions is related to the clustering coefficient in communication networks and the other to the size of a cluster. All four topological summary statistics are used to quantitatively compare in plots and in a least-square approach experimental data to cluster aggregation simulations with decreasing probabilities of aggregation. We study scanning transmission electron micrographs and utilized the intensity - mass thickness relation present in such images to create comparable micrographs from three-dimensional simulations. Finally, a characterization of colloidal silica aggregates and simulated structures is obtained, which allows for an evaluation of the cluster aggregation process for different aggregation scenarios. As a result, we find that the RLCA process fits the experimental data better than the DLCA process.
\end{abstract}

Keywords: Cluster analysis, micrographs, nanoparticle aggregation, replicated point patterns

\footnotetext{
*Corresponding author: Henrike Häbel, Mathematical Sciences, Chalmers University of Technology and the University of Gothenburg, SE-412 96 Göteborg, Sweden; e-mail: henrike.habel@chalmers.se; telephone: +46317725380
} 


\section{Introduction}

The study of irreversible aggregation of colloids is of interest in many industrial applications such as food (Poon \& Haw, 1997) and drug industry (Parakhonskiy et al., 2014; Sabin et al., 2007), where gels with specific mechanical and permeability properties are sought. A common idea is to start from a colloidal stable dispersion, where the interparticular electrostatic repulsion is strong enough to prevent the particles from aggregating. This repulsive force can be screened by the addition of salt or by adjusting the $\mathrm{pH}$ of the solvent. As a result, the particles may start to aggregate and with time form a gel. In principle, the resulting dynamic behavior of the colloids may be modeled using Derjaguin-Landau-Verwey-Overbeek (DLVO)-theory, where attractive van der Waals-London forces as well as repulsive forces are included. Although the DLVO theory in general gives a physically reasonable model for the stability of colloidal gels, it fails to predict the behavior of silica particles seen in laboratories (Allen \& Matijević, 1969, 1970; Horacio et al., 2006, p.20). The missing features are thought to be a description of solvent layers, hydrophobic bonding and surface charge fluctuations. In addition, the DLVO-theory solely describes the interaction between two spherical colloids. That is why alternative methods have often been used for modeling the dynamic aggregation process.

The two most commonly used models describing colloidal aggregation are diffusion limited cluster aggregation (DLCA), where diffusing colloids aggregate upon collision (Meakin, 1983) and reaction limited cluster aggregation (RLCA), where particles need many collisions before aggregating (Kolb \& Jullien, 1984). These models outline two limiting behaviors of colloids, where 1) no repulsive forces are present and the growth process is dominated by the diffusion coefficient of the colloids (DLCA) and 2) the case where the repulsive forces are strong and dominate the growth process (RLCA). These two models have, although their simplistic form, shown a striking similarity with experimental results obtained using scattering techniques such as light scattering (Lin 
et al., 1989a), dynamic light scattering (Kops-Werkhoven \& Fijnaut, 1981; Lin et al., 1989b), small angle X-ray scattering (Wijnen et al., 1991), microscopy techniques (Liu et al., 1990) and indirect methods such as estimates of permeability (Babu et al., 2008). Both theoretical and experimental investigations have shown that the regimes yield a fractal behavior and that this behavior is universal, in the sense that the same behavior occurs for very different types of colloids (Poon \& Haw, 1997; Lin et al., 1989b; Liu et al., 1990). From an experimental point of view, the aggregation processes are often difficult to monitor: the scattering techniques have the limiting feature that only very dilute systems can be monitored and therefore the dynamic aggregation process is not accessible in the long-time limit. As an alternative, a series of microscopy images can be used, where the colloidal state is captured at multiple time points in order to determine the growth process. This approach suffers, however, from the fact that each image is taken from an individual aggregation process at multiple time points resulting in a very time consuming scheme.

In this work, the colloidal aggregation of nano sized silica particles $(20 \mathrm{~nm})$ at 9 $w t \%$ is studied. Silica particle gels are inorganic, non-toxic gels, for which the gelation time can be tuned to be between minutes and days (Jurlnak \& Summers, 1991). The probability of aggregation is studied from scanning transmission electron microscopy (STEM) micrographs representing the resulting aggregated silica particle gel. An example of such a micrograph is given in Part A of Figure 1. A schematic DLVO potential of spherical nano sized silica particles is presented in Part B. Instead of modeling the full potential, a simplified model is used here, where the potential height corresponds to a probability of aggregation to occur. Section 2 provides more detailed information about the experimental data and cluster aggregation simulations. Section 3.1 introduces the summary functions from spatial statistics used to summarize and quantitatively compare the structure characteristic of micrographs experimentally obtained and of simulated micrographs of DLCA and RLCA structures generated under different aggregation scenarios. Section 3.2 presents the least-square approach taken into ac- 
count for conclusions drawn from all summary statistics combined. The corresponding results are presented and discussed in Sections 4 and 5. As a result, we show that it is not necessary to form a time series of microscopy images in order to study the aggregation process since this information is embedded in the resulting gel network. In this way, features of the dynamic aggregation process may be retrieved from a static micrograph representing the resulting silica particle gel.
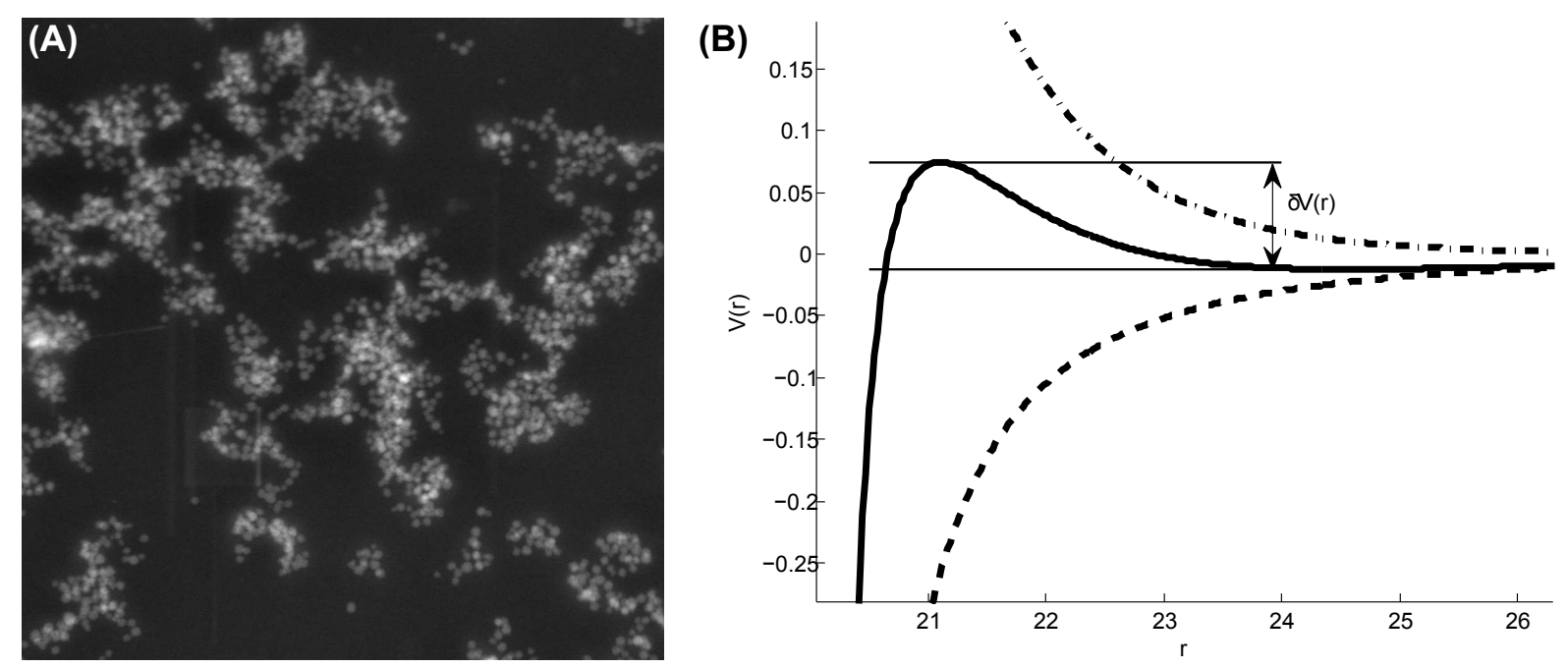

Figure 1: A) STEM micrograph of nano sized silica particle $(20 \mathrm{~nm})$ gel at $9 \mathrm{wt} \%$. B) Schematic DLVO potential (solid) consisting of attractive Van der Waals potential (dashed) and a repulsive electrostatic potential (dashed dotted). The height of the potential barrier $\delta V(r)$ of the diffusing silica spheres influences the resulting configuration of the aggregate. This potential differs from the true effective potential, where also additional effects such as of solvent layers, hydrophobic bonding and surface charge fluctuations are present.

\section{Materials and methods}

\subsection{Colloidal silica gel sample preparation}

The colloidal gel used in this study consisted of an aggregated network of spherical silica nanoparticles $\left(\mathrm{SiO}_{2}\right.$, density $\left.2.2 \mathrm{~g} / \mathrm{cm}^{3}\right)$, where the particles had a diameter of around $20 \mathrm{~nm}$, and the pores were filled with a salt solution. Gels were made from 
charged stabilized particle dispersions, where the negative charges originated from the partly deprotonated hydroxyl groups on the silica surface. Following the destabilization of the dispersion, hydroxyl groups on the particle surfaces chemically combine and form covalent siloxane bonds, resulting in particle aggregates, and finally gelation when these combine to form one large aggregate that spans the entire volume of the sample (Schantz Zackrisson et al., 2006). Siloxane bonds and the mechanical properties of the silica spheres are responsible for the relative rigidity of these gels, making them amenable to sample preparation and STEM imaging without any significant changes to the network structure. A detailed description of the gel synthesis has been reported earlier in Nordin et al. (2014). An aqueous silica dispersion (Bindzil 40/130, 18.2 vol\% silica) was kindly provided by AkzoNobel Pulp and Performance Chemicals, Sweden. The dispersion was filtered to remove larger particle aggregates and the $\mathrm{pH}$ was set to 7.8 by ion exchange. The silica dispersion was mixed with deionized water, sodium chloride solution, vortexed and left undisturbed to gel for 14 days.

\subsection{Image data preparation}

The gel embedding and sectioning prior to the STEM imaging is described in detail in Nordin et al. (2014). In summary, gel cubes extracted from the inner volume of the gel samples were dehydrated in a graded ethanol series and thereafter embedded in LR White resin (TAAB laboratories, Equipment Ltd., Berkshire, UK). Ultra thin sections of about $90 \mathrm{~nm}$ were generated using a diamond knife (DiATOME, Biel, Switzerland) with an ultramicrotome (Powertome XL, RMC products, Boeckeler Instruments Inc, Tucson, Arizona). The sections were imaged using a high angular annular dark-field (HAADF) detector in a Tecnai G2 (FEI Company, Eindhoven, the Netherlands) using an accelerating voltage of $200 \mathrm{kV}$ and a camera length of $300 \mathrm{~mm}$, giving the HAADF detector an inner radius of $22 \mathrm{mrad}$. The obtained STEM micrographs were of a size of 2140 by $2140 \mathrm{~nm}^{2}$ and partitioned into 5 quadratic subwindows with a edge length of 
$600 \mathrm{~nm}$. These subwindows were taken in a chess-like pattern to reduce correlations between them.

\subsection{Cluster aggregation simulations}

In a study of the probability of aggregation assumed to drive the aggregation dynamics in the gel samples studied, the silica particle aggregation process was simulated under different aggregation scenarios. Single particles were randomly placed in a periodic simulation box according to a given volume fraction and were let to diffuse by Brownian motion forming clusters with time. A cluster of particles can consist of one or several aggregated spheres, where the cluster size determines the diffusion coefficient. Upon collision between two clusters, the colliding particles have the possibility of aggregating with probability $p$. A probability $p=1$ gives a growth process in the DLCA regime while $p$ approaching zero gives an RLCA process. If no aggregation occurs, the particles are let to collide elastically. Upon aggregation the collision point is detected and the two clusters merge into one larger cluster. This procedure continues until one sole cluster remains. Rotational diffusion of the clusters and gravitational forces are neglected. Using the same volume fraction as in the gel samples of $5.96 \%$ in a $700 \times 700 \times 700 \mathrm{~nm}^{3}$ box and setting the sphere diameter to $20 \mathrm{~nm}$, a fixed number of 4881 particles was used in the simulations. In total, five simulated aggregated structure were generated with five different probabilities of aggregation $p \in\{1,0.1,0.01,0.001,0.0001\}$. Henceforth, these simulated structures are referred to as cluster aggregation simulations. Two examples of resulting clusters are shown in Figure 2 for a DLCA simulation with $p=1$ and an RLCA simulation with $p=0.0001$. Figure 2 also presents how the simulations were prepared for the data analysis as described in Section 2.4. 

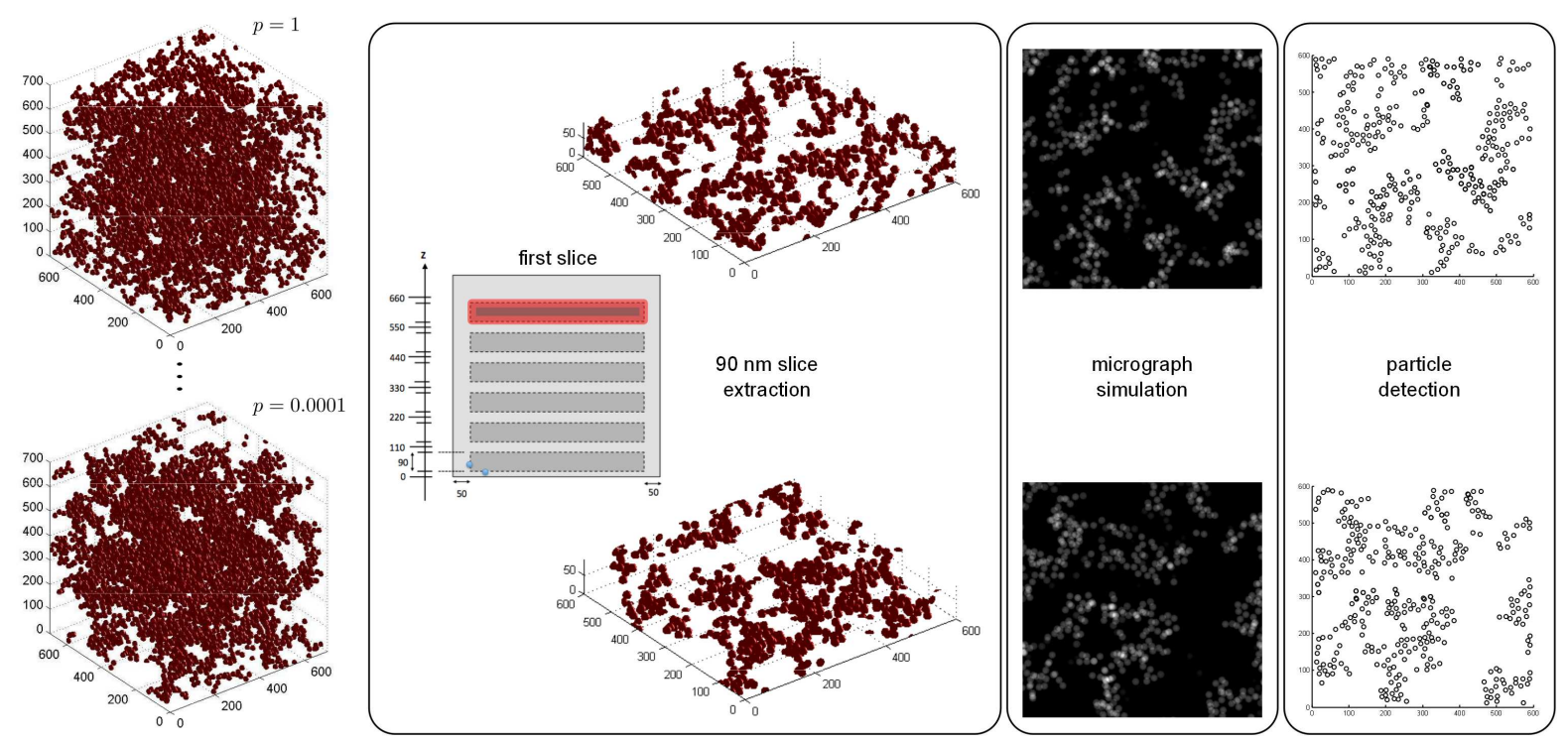

Figure 2: Diagram of data preparation on the example of two cluster aggregation simulations with probabilities of aggregation $p=1$ (top row) and $p=0.0001$ (bottom row), respectively showing three steps: 1) $90 \mathrm{~nm}$ thick slice extraction from the inner $700^{3}$ $\mathrm{nm}^{3}$ cube, 2) micrograph simulation and 3) particle detection. Steps 1)-3) were repeated for six slices per cluster aggregation simulation.

\subsection{Image analysis}

\subsubsection{Simulation of micrographs}

To ensure a fair comparison between two-dimensional experimental data and threedimensional simulated particle aggregates, gray-scale images mimicking STEM micrographs were created from the cluster aggregation simulations. In particular, a central cube with an edge length of $600 \mathrm{~nm}$ was extracted from each generated cluster aggregation simulation. The outer part within each box was excluded from the analysis in order to avoid any wrapping effects due to the usage of a periodic simulation box. The remaining central cube was partitioned into six $90 \mathrm{~nm}$ thick non-overlapping slices as depicted in Figure 2. As a result, six approximately independent repetitions are obtained per cluster aggregation simulation. The corresponding micrographs were formed using the method presented in Nordin et al. (2014) describing the expected intensity 
$I(x, y)$ of a pixel at $(x, y)$ as

$$
I(x, y)=\frac{\int_{0}^{90} \rho_{s}(x, y, z) d z}{90},
$$

where $\rho_{s}$ denotes a mass-intensity function contained in the small pixel region at $(x, y)$. Furthermore, zero-mean Gaussian noise with constant variance, $\sigma^{2}=0.001$, was added to the double gray-scale images with pixel values in $[0,1]$ making the particle detection comparably challenging for simulated as for STEM micrographs.

In the data analysis, the initial, static state of single particles in the simulations prior to diffusion was used as a reference particle distribution. Whereas the completely spatially random case, the Poisson point process, is commonly used as a reference process, a Strauss process (Stoyan et al., 1995, p. $171 \mathrm{ff}$.) was used here. The interaction parameter was set to $10^{-11}$ such that configurations close to a hard-core pattern with no overlapping particles are obtained. In particular, six independent realizations of the hard-core model were generated. The simulations were run with a fixed number of 4881 points in a $700^{3} \mathrm{~nm}^{3}$ observation window which corresponds to the same silica volume fraction as in the gel samples and cluster aggregation simulations. Subsequently, micrographs were also simulated for a single, randomly placed $90 \mathrm{~nm}$ thick slice per reference realization.

\subsubsection{Particle detection}

Tools from image analysis implemented essentially in the Image Processing Toolbox in MATLAB R2013b were used for the particle detection in experimental and simulated micrographs. The particle detection was based on several steps. Prior to particle detection, the micrographs were roughly segmented into empty space and silica phase. Consecutively, each resulting binary image was smoothened with a median filter with a 3 by 3 structure matrix. Then, the silica phase was dilated by a circular structure element of radius $3 \mathrm{~nm}$ in order to facilitate the particle detection. The resulting template 
was subtracted from the original image, such that a gray-scale input image with even background and sharper edges around the silica phase was obtained. Next the particles were detected using the circular Hough transform (Atherton \& Kerbyson, 1999) calculated from pixels above a certain threshold determined by the gradient field of the input image (Peng, 2005). Since this routine searches for circular objects in the input image, some detected round objects were removed as falsely detected particles. The remaining particles were subtracted from the input image and the two particle detection steps, finding round objects and deleting falsely detected particles, were repeated. A comparison of the known number of artificial particles and the number of detected particles in the simulated micrographs showed that on average almost $70 \%$ of the particles were detected and included in the spatial statistical analysis.

\section{Spatial statistical analysis}

For the spatial statistical analysis, the software $R$ version 3.1.0. and especially the $R$ package spatstat version 1.37-0 (Baddeley \& Turner, 2005) was used. In the analysis, a set of detected particle centers was interpreted as a realization of a point process

$$
X=\left\{X_{i}\right\}_{i \in \mathbb{N}}, \mathbb{N}=\{1,2, \ldots\}
$$

Here, $X$ can here be defined as a sequence of random two-dimensional vectors representing the coordinates of the particle centers (Stoyan et al., 1995, p.99 ff.). Henceforth, $X$ is assumed to be invariant under translation and rotation. Since the particles were assumed to be monodispersed with a fixed radius of about $10 \mathrm{~nm}$, the spatial statistical analysis was only conducted on the particle center point patterns disregarding the particle radii. 


\subsection{Structure characterization}

The important characteristics of the experimental and simulated aggregates were investigated using summary functions from spatial statistics assuming stationarity and isotropy. First, the empty space function $F$ was chosen as a first-order characteristic describing the pore space since the pore or empty space is of interest for the properties and functions of gels. Second, Ripley's $K$-function was considered as a secondorder characteristic. These two characteristics are commonly used to test against the completely spatially random case, the Poisson point process. Here, however, different cluster point processes were only compared to each other without testing for a certain distribution. That is why the so called clustering function $c$ as introduced in Rajala (2010) was used to study the inner cluster behavior on a higher-order. In addition, a new summary function based on the diameter of gyration of clusters is introduced, which takes the average of all distances between particles in a cluster into account.

\subsubsection{First-order characteristic}

The empty space function $F:[0, \infty) \longrightarrow[0,1]$ is a morphological summary of the distance distribution of an arbitrary fixed point to the nearest point in $X$ in a micrograph $W \in \mathbb{R}^{2}$. Considering the probability that a ball $b(x, r)$ centered at an arbitrary point $x$ in $W$ with radius $r$ does not contain any point of $X$, a cumulative distribution as a function of radius $r$ is obtained by (Illian et al., 2008, p.201 f.)

$$
F(r)=1-\mathbb{P}(X \cap b(x, r)=\emptyset) \quad \forall r \geq 0 .
$$

In order to deal with the edge effects caused by missing observations beyond the boundaries of $W$, a Kaplan-Meier estimator based on observed points $X \cap W$ was 
used as suggested in Baddeley \& Gill (1997) by

$$
\widehat{F}(r)=1-\exp \left(-\int_{0}^{r}\left(\frac{h_{1}\left(\partial\left(X_{\oplus s}\right) \cap W_{\ominus s}\right)}{\nu_{2}\left(W_{\ominus s} \backslash X_{\oplus s}\right)}\right) d s\right) \quad \forall r \geq 0
$$

for Minkowski operations $X_{\oplus r}=X \oplus b(o, r), W_{\ominus r}=W \ominus b(o, r)$. Here, $h_{1}$ gives the length of the boundary of $X_{\oplus r}$ contained in the eroded window $W_{\ominus r}$ and $\nu_{2}$ the area in this eroded window not occupied by the dilated points $X_{\oplus r}$.

\subsubsection{Second-order characteristic}

Ripley's $K$-function $K:[0, \infty) \longrightarrow[0, \infty]$ was considered here as a second-order characteristic, which is defined by

$$
K(r)=\lambda^{-1} \mathbb{E}_{o}\left(h_{0}(X \cap b(o, r) \backslash\{o\})\right) \quad \forall r \geq 0,
$$

where $h_{0}(A)$ gives the number of points of $X$ in $A \in \mathbb{R}^{2} . \lambda K(r)$ refers to the expected number of further points within distance $r$ of a typical point $o$ of $X$ conditioning that such a point exists (Illian et al., 2008, p.214 ff.). $\lambda$ denotes the intensity, which is the mean number of points of the process $X$ per unit area. Using Ripley's isotropic edge correction, the following estimator for $K$ is obtained for interpoint distances $d_{i j}=$ $\left\|x_{i}-x_{j}\right\|$ by

$$
\widehat{K}(r)=\frac{\nu_{2}(W)}{n(n-1)} \sum_{i=1}^{n} \sum_{j \neq i} w_{i j}^{-1} \mathbb{I}\left(d_{i j}<r\right),
$$

where $n=h_{0}(X \cap W)$ and $\mathbb{I}(\cdot)$ denotes the indicator function with weights

$$
w_{i j}=\frac{h_{1}\left(\partial b\left(x_{i}, d_{i j}\right) \cap W\right)}{h_{1}\left(\partial b\left(x_{i}, d_{i j}\right)\right)} .
$$

Here, $h_{1}$ gives the length of the arc of the ball $b\left(x_{i}, d_{i j}\right)$ lying in $W$, such that $w_{i j}=1$ if $b\left(x_{i}, d_{i j}\right) \subset W$. Detailed calculations can be found in (Diggle, 2014, p.66). Instead of analyzing the $K$-function directly, a variance stabilizing transform, the $L$-function 
$L:[0, \infty) \longrightarrow[0, \infty)$ given by

$$
L(r)=\sqrt{\frac{K(r)}{\pi}} \quad \forall r \geq 0
$$

was considered. Based on the estimator $\widehat{K}$, a plug-in estimator $\widehat{L}$ for the $L$-function was obtained.

\subsubsection{Third-order characteristic}

Intended as a third-order characteristic, the clustering function as introduced in Rajala (2010) and implemented in the R package SGCS Rajala (2014) is used to identify high density groups of points by taking point triplets into account. In particular, the clustering function $c:[0, \infty) \longrightarrow[0,1]$ sets the number of triplets in $b(o, r)$ in relationship to the theoretical number of possible triplets. Hence, it is defined as $c(r)=\mathbb{E}_{o} c_{o, r}$ with

$$
c_{o, r}=\left\{\begin{array}{lll}
\frac{\sum_{j, k: x_{j}, x_{k} \in b(o, r) \backslash\{o\}} \mathbb{I}\left(d_{j k} \leq r\right)}{\frac{1}{2}\left(\delta(o)^{2}-\delta(o)\right)} & , \delta(o) \geq 2 & \\
0 & , \delta(o)<2 &
\end{array}\right.
$$

and $\delta(o)=\sum_{j=1}^{n} \mathbb{I}\left(0<\left\|o-x_{j}\right\| \leq r\right)$. A minus-sampling scheme was applied in order to get an edge corrected estimator

$$
\widehat{c}(r)=\frac{1}{n_{\ominus}} \sum_{i=1}^{n} \mathbb{I}\left(x_{i} \in W_{\ominus b(o, r)} \frac{\sum_{j, k: x_{j}, x_{k} \in b\left(x_{i}, r\right) \backslash\left\{x_{i}\right\}} \mathbb{I}\left(d_{j k} \leq r\right)}{\frac{1}{2}\left(\delta\left(x_{i}\right)^{2}-\delta\left(x_{i}\right)\right)}, \quad \delta\left(x_{i}\right) \geq 2\right.
$$

and 0 otherwise, where $n_{\ominus}=h_{0}\left(X \cap W_{\ominus b(o, r)}\right)$ is the number of points in the eroded window $W_{\ominus b(o, r)}$. 


\subsubsection{Higher-order characteristic}

The topology of silica aggregates can also be characterized by the average cluster size based on the diameter of a cluster

$$
D=2 \sqrt{\frac{1}{2 n_{k}^{2}} \sum_{i=1}^{n_{k}} \sum_{j=1}^{n_{k}} d_{i j}^{2}},
$$

where $n_{k}$ is the number of particles in a cluster. $D$ is also called the diameter of gyration (Khan et al., 2014). In this study, the samples taken and especially the micrographs obtained do not show that the nanoparticle gel consists of one large cluster. Instead smaller clusters are visible and the question of how to discriminate them from each other arises. Here, geometric graphs (Penrose, 2003) placing an edge between two points if $d_{i j} \leq r$ were used to identify clusters. As a result, clusters are formed by points connected in the graph and the mean cluster size function $M:[0, \infty) \longrightarrow[0, \infty)$ can be defined as a function of $r$ by

$$
M(r)=\mathbb{E}(D(r)) \quad \forall r>0
$$

In order to account for edge effects, a weighted average of the diameter of gyration of $K$ clusters was used for the estimation of $M$ such that

$$
\widehat{M}(r)=\sum_{k=1}^{K} w_{k} D_{k}(r)
$$

with weights $w_{k}=\frac{n_{k}}{n}$ as the proportion of the number $n_{k}$ of points in the $k^{\text {th }}$ cluster to the total number $n$ of points in $W$. The geometric graphs were constructed using the $\mathbf{R}$ package spatgraphs (Rajala, 2012). 


\subsubsection{Handling of replicated point patterns}

Given three times five replicates of STEM micrographs from gel samples and six replicates from each cluster aggregation simulation, related summary functions $f_{i}, i=$ $1, \ldots, I$, where $I$ refers to the number of replicates, were pooled by calculating the weighted averages

$$
\widehat{f}(r)=\frac{\sum_{i=1}^{I} n_{i} \widehat{f}_{i}(r)}{\sum_{i=1}^{I} n_{i},}
$$

where the weights are determined by the number of points $n_{i}$ in the respective point pattern (Diggle, 2014, p.92 f.).

\subsection{Least-squares approach for comparing aggregation scenarios}

The probability of aggregation $p$ assumed to drive the particle aggregation dynamics was studied in a simulation based approach. The aim was to estimate the dynamic aggregation process from static images of completely aggregated structures. In order to identify one group of simulated structures that is most similar to the STEM data, a least-squares approach inspired by Redenbach \& Särkkä (2013) was chosen on a grid of five probabilities of aggregation $p \in\{1,0.1,0.01,0.001,0.0001\}$. In this study, the pooled summary functions of the STEM micrographs $f_{j}$ and the simulated micrographs $\tilde{f}_{i_{p} j}$ per aggregation scenario $i_{p}=1, \ldots, 5$ were compared by calculating the relative squared error at $T \in \mathbb{N}$ chosen equally spaced distances $r_{1}, \ldots, r_{T}$ resulting in sums of squares

$$
S_{i_{p} j}=\frac{1}{T} \sum_{t=1}^{T}\left(\frac{\tilde{f}_{i_{p} j}\left(r_{t}\right)-f_{j}\left(r_{t}\right)}{f_{j}\left(r_{t}\right)}\right)^{2},
$$

for each summary function $j=F, L, c, M$. If the different aggregation scenarios can be discriminated from each other, the probability of aggregation linked to the smallest total sum of squares of the evaluated summary functions

$$
S_{i_{p}}=S_{i_{p} F}+S_{i_{p} L}+S_{i_{p} c}+S_{i_{p} M}
$$


can be associated with the probability of the gel samples. Using relative differences allows here for comparisons of summary functions on different scales.

\section{Data analysis}

\subsection{Summary characteristics}

All summary functions used for the characterization of particle structures in the micrographs are presented in Figure 3, namely the empty space function $F$, the $L$-function, the clustering function $c$ and the mean cluster size function $M$. All cluster aggregation summary functions of first and second-order lie between those of particle patterns prior to (reference) and after aggregation (STEM data). Therefore, the cluster aggregation processes seem to be applicable for modeling the silica particle aggregation dynamics. If the cluster aggregation simulations had not shown a clear difference to the static reference pattern prior to aggregation, the modeled aggregation would have been ineffective and, hence, the corresponding model unfeasible. Moreover, it can be seen in Figure 3 that the lower the probability of aggregation, the more the cluster aggregation summary functions approach the data curves. Hence, it can also be concluded that the DLCA process with probability of aggregation equal to one is the best model for the silica particle aggregation dynamics. Interestingly, there is no clear ranking of the cluster aggregation summary functions according to their probability of aggregation visible for the higher-order functions. However, the lowest probability of aggregation appears to give the best overall result.

Inference on the topology of the particle aggregates can be drawn from each plot in Figure 3. Starting with the first-order characteristic, the empty space function, it appears that there is a larger empty space or space of pores in the gel samples than in the cluster aggregation simulations. One reason for this can be the fact that larger clusters tend to sink to the bottom of the sample due to gravity, an effect not incorpo- 
rated in the cluster aggregation simulations. However, it can be seen that the smaller the probability of aggregation, the larger the empty space. This indicates that the particles aggregate in more round and tighter fashion for small probabilities of aggregation, which is in line with the expected theoretical aggregation behavior. Interestingly, there is almost a perfect ordering of empty space functions according to the respective probability of aggregation. The same can be observed for the $L$-function. Furthermore, one can identify a small change in the slope of the $L$-function at the radius of $20 \mathrm{~nm}$. Since this change is also present for the reference $L$-function, there is some evidence that the particle detection algorithm introduces a hard-core distance between the points which may disguise larger differences between the cluster aggregation simulations at smaller radii. Also in the clustering and the mean cluster size function there is hardly any visible difference between the cluster aggregation simulations up to a radius of $20 \mathrm{~nm}$. In fact, the STEM and cluster aggregation clustering functions are very similar up to a radius of about $50 \mathrm{~nm}$. For larger radii the cluster aggregation simulations tend to deviate from the STEM data indicating a tighter clustering in the gel samples. This conclusion is supported by the results for the mean cluster size function. Here, one can also detect a tighter clustering for the STEM data than for the cluster aggregation simulations. Remarkably, the smaller probabilities of aggregation appear to result in more chain like clusters at small ranges up to $50 \mathrm{~nm}$ than the larger probabilities. In contrast to the DLCA regime and the RLCA simulations with probabilities down to 0.01 , however, the RLCA simulations closest to zero seem to form tighter clusters at larger ranges still showing a smaller mean cluster size than the reference case, where particles did not aggregate.

\subsection{Least-square analysis of the probability of aggregation}

As discussed in Section 4.1, the summary functions for the different aggregation scenarios were not very different from each other. However, there were some indica- 
tions on the probability of aggregation associated with the gel samples. In order to strengthen these conclusions, the integrated relative difference between STEM summary functions and those of the different cluster aggregation simulations was approximated by calculating sums of squares as in equation (1) at $T=40$ equidistant distances between $r_{1}=22$ and $r_{T}=100$. Distances below 22 were not taken into account due to the effect introduced to the point patterns by the particle detection algorithm for distances up to $20 \mathrm{~nm}$ as mentioned in Section 4.1. Table 1 presents the sums of squares per summary function and aggregation scenario: without aggregation (reference), DLCA $(p=1)$ and RLCA approaches ( $p \in\{0.1,0.01,0.001,0.0001\})$. These sums of squares reflect the behavior seen in Figure 3. On trying to identify one probability of aggregation whose corresponding simulated structures are most similar to the STEM data, the first, second and higher-order summary functions, $F, L$ and $M$, suggest $p=0.0001$, but the third-order characteristic, $c$, indicates that $p=0.1$ seems to model the short range repulsive behavior within a cluster best.

Table 1 gives the total sums of squares over all summary function per aggregation scenario presented in Figure 3. Obviously, the cluster aggregation simulations perform better than the simulations without aggregation as models for the silica particle aggregation dynamics since their total sums of squares are only half as large as for the reference scenario. Whereas it is difficult to detect a clear difference between the DLCA regime and the larger probabilities of aggregation $p \in\{0.1,0.01\}$ tested for the RLCA regime, there seems to be a distinct improvement from $p=0.001$ onward. Overall, the smallest probability of aggregation appears to be in closest agreement with the experimental data among the tested probabilities of aggregation, but even a smaller probability seems to be required for capturing the particle aggregation dynamics of the gel samples. 


\begin{tabular}{l|cccc|c}
\hline $\begin{array}{l}\text { Probability of } \\
\text { aggregation }\end{array}$ & \multicolumn{4}{|c|}{ Summary statistics } & Total \\
reference & 0.5154 & 0.1481 & 0.0722 & 0.5189 & 1.2547 \\
$\mathrm{p}=1$ & 0.2928 & 0.0868 & 0.0054 & 0.2258 & 0.6108 \\
$\mathrm{p}=0.1$ & 0.3042 & 0.0908 & 0.0036 & 0.1861 & 0.5847 \\
$\mathrm{p}=0.01$ & 0.2750 & 0.0818 & 0.0044 & 0.2693 & 0.6305 \\
$\mathrm{p}=0.001$ & 0.2635 & 0.0732 & 0.0072 & 0.2183 & 0.5622 \\
$\mathrm{p}=0.0001$ & 0.2420 & 0.0678 & 0.0061 & 0.1429 & 0.4588 \\
\hline
\end{tabular}

Table 1: Comparison of the pooled summary statistics for STEM and simulated cluster aggregation micrographs for the reference structure prior to aggregation and five aggregated structures generated with decreasing probabilities of aggregation

\section{Discussion}

For the spatial statistical analysis, other first and second-order characteristics are available. For instance, another commonly used first-order characteristic is the nearest neighbor distance distribution function. It is important to keep in mind that particles are not overlapping in the three-dimensional structure which would result in a nearestneighbor distance of $20 \mathrm{~nm}$. Projected to micrographs, particles may overlap making a nearest neighbor analysis feasible. Here, however, the hard-core distance identifiable in Figure 3 indicates that the implemented particle detection algorithm induces such a hard-core distance as well in the micrographs. In fact, only around $40 \%$ of particles with a projected interpoint distance of $20 \mathrm{~nm}$ were detected on average. Consequently, the nearest neighbor distance distribution was not used in the analysis since no crucial differences between the different aggregation scenarios were preserved. Also a combination of several $\mathbf{k}^{\text {th }}, k \geq 1$, nearest neighbor distance functions did not appear useful. An alternative second-order summary function related to the $K$-function is the pair correlation function, which is a normalized transform of the derivative of the $K$-function. In order to obtain an estimator for the derivative, kernel smoothing is needed based on a certain bandwidth. The $K$-function was chosen here since no bandwidth had to be specified.

Overall, we had expected to see more prominent differences between the summary 
functions of the different cluster aggregation simulations. In analogous simulation onlattice, where all clusters have the same diffusion coefficient, we found a very clear difference between the different aggregation scenarios. We suspect that either the increased degree of freedom for the clusters using an off-lattice simulation or the lowering of the diffusion coefficient as the clusters grow (or both) have an impact on the results. In the first case, it would be very good to study RLCA simulations with even lower probabilities of aggregation than considered in the study, especially since the data analysis suggests lowering the probability of aggregation as well. Unfortunately, such simulations are not feasible since they demand very high computational effort. Instead, an alternative way for generating such particle aggregates should be developed. It deserves mentioning that in a small simulation based validation study, the three-dimensional cluster aggregation simulations only showed a clear difference between two groups of probabilities of aggregation, namely between $p \in\{1,0.1,0.01\}$ and $p \in\{0.001,0.0001\}$. Furthermore, the behavior of the three-dimensional summary functions was in line with summary functions from the micrographs.

\section{Conclusion}

In this study, we investigated the aggregated structure of nanoparticles gels and the process that creates such structures. In particular, we analyzed two-dimensional STEM micrographs from three-dimensional nano sized silica particle gel samples. In order to understand the aggregation process of the nanoparticles, simulated particle aggregates were generated using the DLCA and RLCA process. Since these processes are driven by the probability of the particles to aggregate upon collision, it was also of interest to approximate this probability of aggregation for the given gel samples. Five decreasing probabilities of aggregation were used in the cluster aggregation simulations. For the comparison of two-dimensional STEM data to three-dimensional simulations, micrographs were simulated from the particle aggregates generated with the 
cluster aggregation processes. For the comparison, summary functions from spatial statistics were used to characterize the different point patterns obtained by detecting the particles in the micrograph with tools from image analysis. Besides commonly used summary statistics, the spatial statistical analysis included also newly developed functions for cluster analysis, where the one based on the diameter of gyration was introduced in this work. The results from both, the visual inspection of the summary functions and the least-square approach, indicated that even smaller probabilities of aggregation should be studied in order to find the one that can be associated with the gel samples. Since simulations with very small probabilities of aggregation require a lot of computations, alternative simulation methods should be developed. Furthermore, there is also some evidence that for modeling the short range inner cluster behavior, larger probabilities of aggregation appear more suitable, such that a hierarchical mixture aggregation process with a change in probability of aggregation could be worth including in such alternative models.

Even though, this study further showed that inference from two-dimensional projections can be drawn on three-dimensional clustered structures, a validation with replicated three-dimensional data should be conducted. Further studies could also include two-dimensional summary functions for marked point processes, where the image intensity at the center of a particle is attached as a mark to each point of the process. It could be tested if intensity based two-dimensional mark summary functions can be used to describe the particle distribution in three dimensions.

All in all, it became evident, that the DLCA process might not be the best choice when modeling silica nanoparticle aggregates and that the RLCA regime should be preferred. Moreover, this study showed that it is possible to draw inference on threedimensional particle aggregation dynamics directly from static two-dimensional micrographs of the resulting gel without requiring a time consuming and complicated time series of microscopy images. 
(A) Empty space function

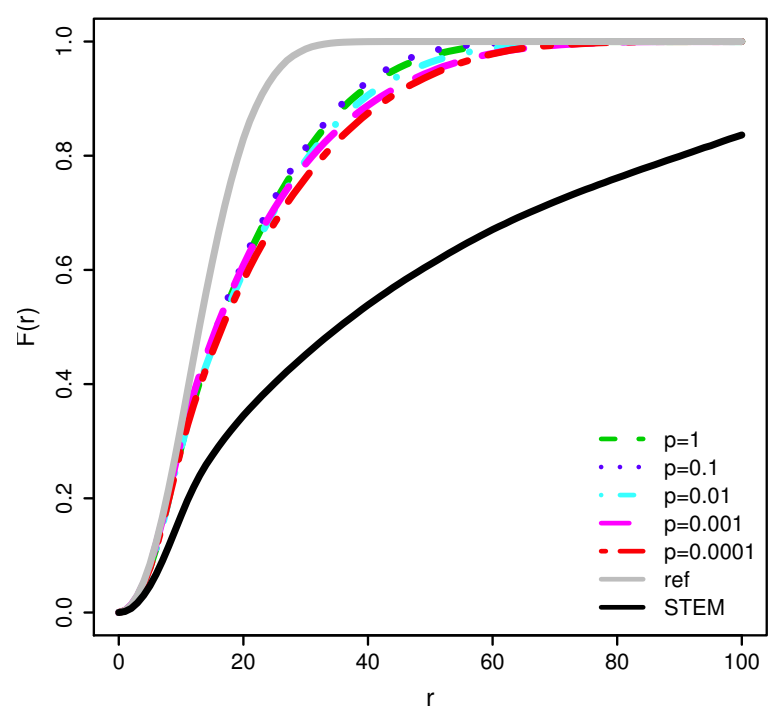

(C) Clustering function

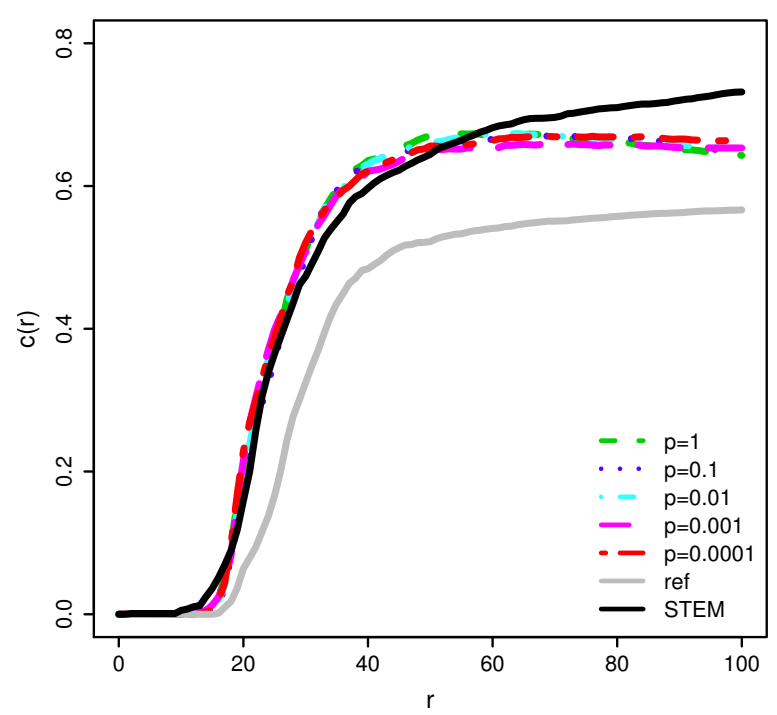

(B) L-function

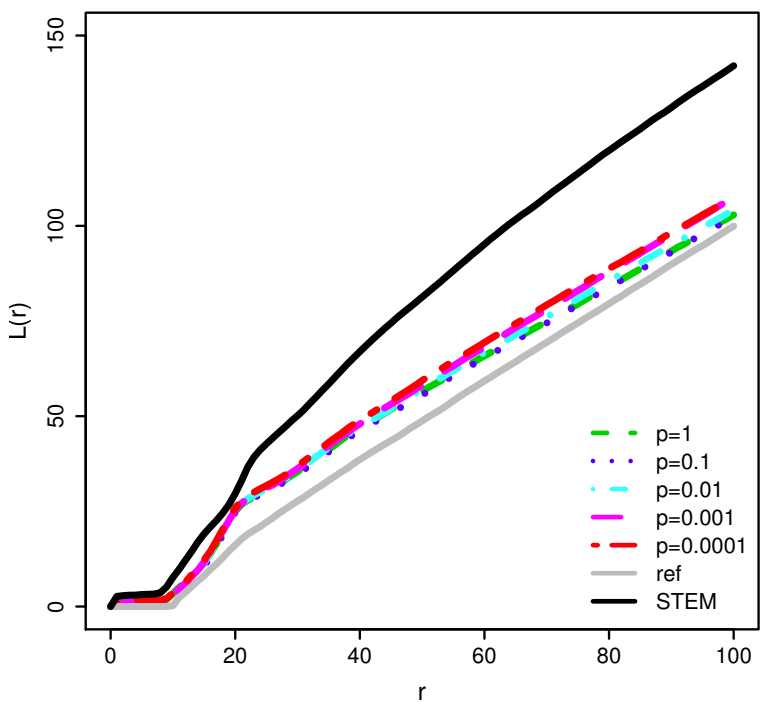

(D) Mean cluster size function

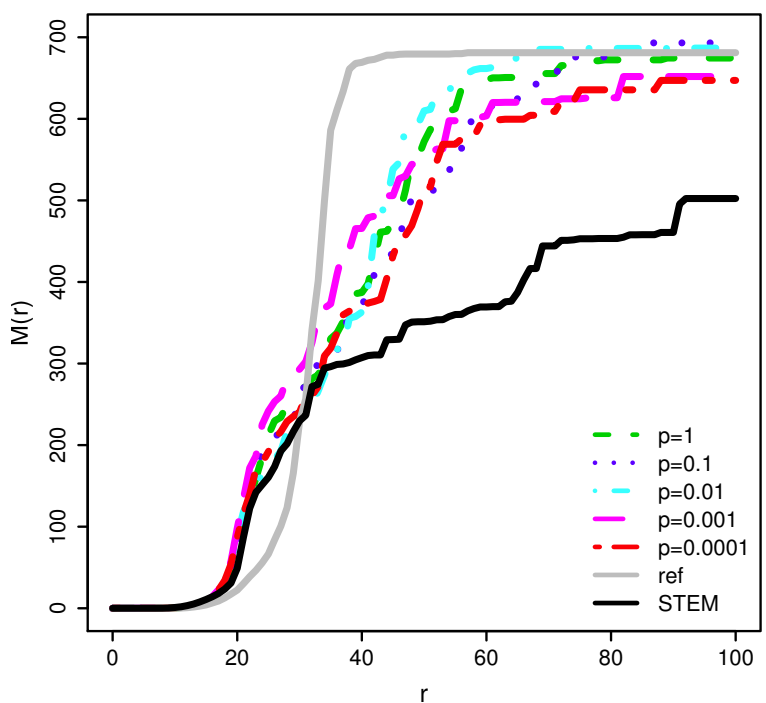

Figure 3: (A) Empty space function $F$, (B) $L$-function, (C) clustering function $c$ and (D) mean cluster size function $M$ pooled for detected point patterns from cluster aggregation simulations with five different aggregation scenarios, realizations of a reference point process without particle aggregation and STEM micrographs. It becomes evident that the process with the smallest probability of aggregation $(p=0.0001)$ tends to describe the aggregation dynamics of silica nanoparticles best. 


\section{Acknowledgments}

This work is part of the VINN Excellence Centre SuMo Biomaterials and has mainly been financed by the Swedish Governmental Agency for Innovations Systems, VINNOVA. In addition, the financial support from the Knut and Alice Wallenberg Foundation, KAW, and the Swedish Foundation for Strategic Research, SSF, is highly appreciated. The authors would also like to thank Mojtaba Siahpoosh for assisting in the gel sample preparation and Tuomas Rajala for valuable discussions as well as contributions to the programming in $\mathrm{R}$.

\section{References}

Allen, L. \& Matijević, E. (1969). Stability of colloidal silica: I. Effect of simple electrolytes, Journal of Colloid and Interface Science, 31(3):287 - 296. doi: 10.1016/0021-9797(69)90172-6.

Allen, L. \& Matijević, E. (1970). Stability of colloidal silica: II. Ion exchange, Journal of Colloid and Interface Science, 33(3):420 - 429. doi: 10.1016/0021-9797(70)902341.

Atherton, T. \& Kerbyson, D. (1999). Size invariant circle detection, Image and Vision Computing, 17:795-803. doi: 10.1016/S0262-8856(98)00160-7.

Babu, S., Gimel, J., \& Nicolai, T. (2008). Tracer Diffusion in Colloidal Gels, The Journal of Physical Chemistry B, 112(3):743-748. doi: 10.1021/jp076342+.

Baddeley, A. \& Gill, R. (1997). Kaplan-Meier estimators of interpoint distance distributions for spatial point processes, The Annals of Statistics, 25(1):263-292. doi: doi:10.1214/aos/1034276629. 
Baddeley, A. \& Turner, R. (2005). spatstat: An R Package for Analyzing Spatial Point Patterns, Journal of Statistical Software, 12(6):1-42.

Diggle, P. (2014). Statistical analysis of spatial and spatio-temporal point patterns. CRC Press, Taylor \& Francis Group, Boca Raton, 3 edition.

Horacio, E., Bergna, E., \& Roberts, W. (2006). Colloidal silica: fundamentals and applications. CRC Press, Boca Raton.

Illian, J., Penttinen, A., Stoyan, H., \& Stoyan, D. (2008). Statistical Analysis and Modelling of Spatial Point Patterns. John Wiley and Sons, Ltd, Chichester.

Jurlnak, J. \& Summers, L. (1991). Oilfield Applications of Colloidal Silica Gel, SPE Production Engineering, pages 406-412. doi: 10.2118/18505-PA.

Khan, M. N., Auerbach, S., \& Monson, P. (2014). Lattice model for silica polymerization: Monte Carlo simulations of the transition between gel and nanoparticle phases, The Journal of Physical Chemistry. B, 118(37):10989-99. doi: 10.1021/jp504961q.

Kolb, M. \& Jullien, R. (1984). Chemically limited versus diffusion limited aggregation, J. Physique Lett., 45(20):977-981. doi: 10.1051/jphyslet:019840045020097700.

Kops-Werkhoven, M. M. \& Fijnaut, H. M. (1981). Dynamic light scattering and sedimentation experiments on silica dispersions at finite concentrations, The Journal of Chemical Physics, 74(3):1618-1625. doi: 10.1063/1.441302.

Lin, M. Y., Lindsay, H. M., Weitz, D. A., Ball, R. C., Klein, R., \& Meakin, P. (1989). Universality of Fractal Aggregates as Probed by Light Scattering, Proceedings of the Royal Society of London A: Mathematical, Physical and Engineering Sciences, 423 (1864):71-87. doi: 10.1098/rspa.1989.0042.

Lin, M. Y., Lindsay, H. M., Weitz, D. A., Ball, R. C., Klein, R., \& Meakin, P. (1989). Universality in colloid aggregation, Nature, 339(6223):360-362. doi: 10.1038/339360a0. 
Liu, J., Shih, W., Sarikaya, M., \& Aksay, I. (1990). Fractal colloidal aggregates with finite interparticle interactions: Energy dependence of the fractal dimension, Physical Review A, 41(6):3206-3215. doi: 10.1103/PhysRevA.41.3206.

Meakin, P. (1983). Formation of Fractal Clusters and Networks by Irreversible Diffusion-Limited Aggregation, Physical Review Letters, 51(13):1119-1122. doi: 10.1103/PhysRevLett.51.1119.

Nordin, M., Abrahamsson, C., Hamngren Blomqvist, C., Häbel, H., Röding, M., Olsson, E., Nydén, M., \& Rudemo, M. (2014). Estimation of mass thickness response of embedded aggregated silica nanospheres from high angle annular dark-field scanning transmission electron micrographs, Journal of Microscopy, 253(2):166-70. doi: 10.1111/jmi.12107.

Parakhonskiy, B., Yashchenok, A., Konrad, M., \& Skirtach, A. (2014). Colloidal microand nano-particles as templates for polyelectrolyte multilayer capsules, Advances in Colloid and Interface Science, 207:253 - 264. doi: 10.1016/j.cis.2014.01.022.

Peng, T. (2005). Detect circles with various radii in grayscale image via Hough Transform, MATLAB Central File Exchange, updated November 17, 2010.

Penrose, M. (2003). Random Geometric Graphs. Oxford University Press, Oxford.

Poon, W. \& Haw, M. (1997). Mesoscopic structure formation in colloidal aggregation and gelation, Advances in Colloid and Interface Science, 73:71 - 126. doi: 10.1016/S0001-8686(97)90003-8.

Rajala, T. (2010). Spatial point processes and graph based statistical features, 2000 Mathematics Subject Classification, Preprint 385.

Rajala, T. (2012). spatgraphs: Graphs for spatial point patterns. URL http: //CRAN.R-project.org/package=spatgraphs. R package version 2.62. 
Rajala, T. (2014). SGCS: Spatial Graph based Clustering Summaries for spatial point patterns. URL http://CRAN. R-project.org/package=SGCS. R package version 2.0.

Redenbach, C. \& Särkkä, A. (2013). Parameter estimation for growth interaction process using spatio-temporal information, Computational Statistics and Data Analysis, 25(1):263-292. doi: 10.1016/j.csda.2012.08.006.

Sabin, J., Prieto, G., Ruso, J., \& Sarmiento, F. (2007). Fractal aggregates induced by liposome-liposome interaction in the presence of Ca2+, The European Physical Journal E, 24(2):201-210. doi: 10.1140/epje/i2007-10231-3.

Schantz Zackrisson, A., Martinelli, A., Matic, A., \& Bergenholtz, J. (2006). Concentration effects on irreversible colloid cluster aggregation and gelation of silica dispersions, Journal of Colloid and Interface Science, 301(1):137-44. doi: 10.1016/j.jcis.2006.04.066.

Stoyan, D., Kendall, W., \& Mecke, J. (1995). Stochastic Geometry and its Applications. John Wiley and Sons, Ltd, Chichester, 2 edition.

Wijnen, P. W. J. G., Beelen, T. P. M., Rummens, K. P. J., Saeijs, H. C. P. L., \& van Santen, R. A. (1991). Silica gel from water glass: a SAXS study of the formation and ageing of fractal aggregates, Journal of Applied Crystallography, 24(5):759-764. doi: $10.1107 /$ S0021889891000924. 